CLINICAL STUDY

\title{
Non-diabetic metabolic syndrome and obesity do not affect serum paraoxonase and arylesterase activities but do affect oxidative stress and inflammation
}

\author{
Suzan Tabur ${ }^{1}$, Ayse Nur Torun ${ }^{1}$, Tevfik Sabuncu ${ }^{1}$, Mehmet Nuri Turan $^{2}$, Hakim Celik ${ }^{3}$, Ali Ruza Ocak ${ }^{3}$ \\ and Nurten Aksoy ${ }^{3}$ \\ Departments of ${ }^{1}$ Endocrinology and Metabolic Diseases, ${ }^{2}$ Internal Medicine and ${ }^{3}$ Clinical Biochemistry, Harran University School of Medicine, \\ 63300 Sanliurfa, Turkey \\ (Correspondence should be addressed to A N Torun; Email: aysenurizol@yahoo.com)
}

\begin{abstract}
Objective: Paraoxonase-1 (PON-1), which has PON and arylesterase activities, is a high-density lipoprotein (HDL)-bound antioxidant enzyme that inhibits atherosclerosis. Diabetes has been shown to have an impact on oxidative stress. The effect of metabolic syndrome (MetS) on oxidative stress and PON-1 has been shown before, and PON-1 has been found to be related with accelerated atherogenesis. This study aimed to determine the oxidative state and PON and arylesterase activities in non-diabetic MetS and non-MetS obese patients.

Design: Thirty obese patients ( $3 \mathrm{M}$ and $27 \mathrm{~F}$ ) without MetS, 40 non-diabetic obese patients ( $3 \mathrm{M}$ and 37 F) with MetS, and 30 controls ( $2 \mathrm{M}$ and $28 \mathrm{~F}$ ) were enrolled.

Methods: A $75 \mathrm{~g}$ glucose tolerance test was performed. PON-1, PON, arylesterase, total antioxidant status (TAS), high-sensitive C-reactive protein (hsCRP), and metabolic parameters were analyzed.

Results: PON and arylesterase activities were similar between the groups, while TAS was low in both MetS and obese groups compared to controls $(P<0.01$ and $P<0.05$ respectively). CRP was higher in the MetS group compared with the obese and control groups $(P<0.01$ and $P<0.001$ respectively). In both the obese and MetS groups, CRP showed a positive correlation with body mass index (BMI). TAS was negatively correlated with BMI, waist circumference, triglyceride levels, and systolic and diastolic blood pressures $(P<0.001)$.

Conclusions: Oxidative stress is altered in non-diabetic MetS and non-MetS obese patients, but PON and arylesterase activities seem not to be affected. This result may be due to the absence of diabetes, the most severe form of altered carbohydrate metabolism.
\end{abstract}

European Journal of Endocrinology 162 535-541

\section{Introduction}

Paraoxonase-1 (PON-1), which has both PON and arylesterase activities, is a high-density lipoprotein (HDL)-associated enzyme that protects low-density lipoprotein cholesterol (LDL-C) and HDL cholesterol (HDL-C) against oxidative damage, and these activities have been found to be inversely related to atherosclerosis (1-3).

Metabolic syndrome (MetS) is defined as the existence of obesity, insulin resistance, glucose intolerance, hypertension, and dyslipidemia (4). Subjects with MetS may be obese but all obese patients may not have MetS. Both MetS and obesity have been shown to have impacts on cardiovascular mortality and morbidity (5). Several reports have indicated that MetS and obesity may alter oxidative stress, which contributes to atherosclerosis-related cardiovascular events $(6,7)$.
However, these studies did not exclude diabetes, which severely affects oxidative stress and atherosclerosis. Therefore, we aimed to demonstrate overall and HDL-related antioxidant status in non-diabetic MetS and only obese patients.

\section{Materials and methods}

\section{Subjects}

Patients who were admitted for the evaluation of obesity were recruited from the Endocrinology and Internal Medicine outpatient clinic. A standard $75 \mathrm{~g}$ oral glucose tolerance test (OGTT) was administered to all participants, and patients were randomized to three groups according to their affected glucose metabolism. Groups included 30 obese patients without MetS and 
glucose intolerance (mean age $31.5 \pm 6.2$ years, $3 \mathrm{M}$ and $27 \mathrm{~F}), 40$ non-diabetic obese patients with MetS (mean age $34.7 \pm 7.2$ years, $3 \mathrm{M}$ and $37 \mathrm{~F}$ ), and 30 volunteer control subjects who were recruited from the Internal Medicine Department (mean age 33.1 \pm 7.8 years, $2 \mathrm{M}$ and $28 \mathrm{~F}$ ). Although the MetS group was composed of non-diabetics, all the patients except one had varying degrees of glucose intolerance or were insulin resistant. The control group had normal OGTT. MetS is defined according to the criteria accepted in the Third Report of the National Cholesterol Education Program (NCEP) (4). Hypertension and hyperlipidemia were diagnosed for the first time at the initiation of the study, so no participant was using an anti-hypertensive or anti-lipidemic drug before obtaining the blood samples. Age, weight, height, body mass index (BMI: body weight $(\mathrm{kg}) /$ height $\left.(\mathrm{cm})^{2}\right)$, and systolic (SBP) and diastolic blood pressures (DBP) of all subjects were recorded. Fasting plasma glucose (FPG), plasma glucose following $75 \mathrm{~g}$ glucose administration, HDL-C, LDL-C, total cholesterol, triglyceride (TG), high-sensitive C-reactive protein (hsCRP), total antioxidant status (TAS), total oxidative status (TOS), oxidative stress index (OSI), and arylesterase and PON activities of all subjects were analyzed. The study was approved by the local ethics committee, and all participants gave signed informed consent.

\section{Blood samples and preparation}

Blood samples were drawn after overnight fasting, and serum samples were stored at $-80^{\circ} \mathrm{C}$ until biochemical determination of TAS, PON, and arylesterase activities.

\section{Measurement of total antioxidant status}

Serum TAS was determined using a novel automated measurement method developed by Erel (8). In the method, hydroxyl radical, the most potent biological radical, is produced first. In the assay, reagent 1 containing ferrous ion solution is mixed with reagent 2 , which contains hydrogen peroxide. The sequentially produced radicals, such as brown colored dianisidinyl radical cation produced by the hydroxyl radical, are also potent radicals. The anti-oxidative effect of the study sample against the potent-free radical reactions, which are initiated by the produced hydroxyl radical, is measured. The assay has excellent precision values, lower than 3\%, and the results are expressed as mmol Trolox Equiv./l.

\section{Measurement of total oxidant status}

Serum TOS was determined using a novel automated measurement method developed by Erel (9). Oxidants present in the study sample oxidize the ferrous ion-o-dianisidine complex to ferric ion. The oxidation is enhanced by glycerol molecules, which are abundantly present in the reaction medium. The ferric ion makes a colored complex with xylenol orange in an acidic medium. The color intensity, which can be measured spectrophotometrically, is related to the total amount of oxidant molecules present in the sample. The assay is calibrated with hydrogen peroxide, and the results are expressed as $\mu \mathrm{mol} \mathrm{H}_{2} \mathrm{O}_{2}$ Equiv./l.

Oxidative stress index Percent ratio of TOS to TAS level was accepted as OSI (OSI (Arbitrary Unit) $=$ TOS ( $\mu \mathrm{mol} \mathrm{H}_{2} \mathrm{O}_{2}$ Equiv./l)/TAS (mmol Trolox Equiv./l)) (10).

\section{Measurements of PON and arylesterase activities}

PON and arylesterase activities were measured with commercially available kits (Relassay, Gaziantep, Turkey). PON measurement was performed either in the presence (salt-stimulated) or in the absence of $\mathrm{NaCl}$. Paraoxon hydrolysis rate (diethyl-p-nitrophenyl phosphate) was measured by monitoring increased absorption at $412 \mathrm{~nm}$ at $37^{\circ} \mathrm{C}$. The amount of generated $p$-nitrophenol was calculated from the molar absorption coefficient at $\mathrm{pH} 8.5$, which was $18.290 / \mathrm{M}$ per $\mathrm{cm}$ (11). PON activity was expressed as U/l serum. The coefficient of variation $(\mathrm{CV})$ for individual samples was $1.8 \%$. Arylesterase activity was measured using phenyl acetate as substrate. Enzymatic activity was calculated from the molar absorption coefficient of the produced phenol, 1310/M per $\mathrm{cm}$. One unit of arylesterase activity was defined as $1 \mu \mathrm{mol}$ phenol generated per minute under the above conditions and expressed as U/l (12). The CV for individual serum samples was $4.1 \%$. The sensitivities of both tests were over $98 \%$.

Plasma TG, total cholesterol, LDL-C, and HDL-C concentrations were measured by automated chemistry analyzer (Aeroset, Abbott) using commercial kits (Abbott). Serum hsCRP level was measured using an available commercial kit (Roche). Insulin resistance was estimated with homeostasis model assessment insulin resistance index (HOMA-IR), and HOMA-IR > 2.29 was accepted as indicating insulin resistance $(13,14)$. Patients with diabetes, active infection, current smoking history, systemic disorder, or drug use affecting the oxidant state or lipid parameters (e.g. statins, angiotensin converting enzyme inhibitors, anti-inflammatory drugs, and oral contraceptives) were excluded.

\section{Statistical analysis}

All analyses were conducted using SPSS 11.5 (SPSS for Windows 11.5, Chicago, IL, USA). Continuous variables were expressed as mean \pm s.D., and non-parametric data were expressed as median and ranges. One-way ANOVA was used for multiple comparisons among the groups, and the Tukey HSD test was used if any statistical 
significance was found. Normality of distribution was evaluated with the Kolmogorov-Smirnov test. Pearson correlation test was used to evaluate any relationships between parameters. Multiple linear regression analysis was done to define the variables that may affect PON and arylesterase. All statistical tests were two-sided, and a $P$ value $<0.05$ was considered significant.

\section{Results}

Mean ages of the three groups were similar. BMI, SBP, DBP, and TG levels were significantly higher in the MetS group compared to the obese group (all $P<0.001$ ). Both obese and MetS groups had significantly lower HDL-C levels than the control group $(P<0.001$ and $P<0.01$ respectively). The obese group had higher BMI and SBP and DBP than the control group (all $P<0.001$ ). LDL-C levels of the MetS and obese groups were similar, but were higher than those of controls (both $P<0.001$ ). All of these clinical and metabolic data are shown in Table 1. In the MetS group, 12 patients had only impaired fasting glucose (IFG) $(12 / 40,30 \%), 1$ had only impaired glucose tolerance (IGT) $(1 / 40,2.5 \%), 10$ had both IFG and IGT (10/40, 25\%), 16 were only insulin resistant $(16 / 40,40 \%)$, and 1 had no abnormal glucose metabolism (1/40, 2.5\%). All patients in the MetS group were hypertensive. Of the 30 obese patients, 24 were insulin resistant, and none of them had abnormal glucose tolerance $(24 / 30,80 \%)$.

hsCRP level was higher in the MetS group compared to both obese and control groups (both $P<0.001$ ), while it was similar in the obese and control groups. TAS was lower in both MetS and obese groups than in the control group $(P<0.001$ and $P<0.05$ respectively). OSI was significantly higher in the MetS group compared to the control group $(P<0.05)$. OSI was also higher in the obese group than in the control group, but the difference was not significant (Table 1). PON and arylesterase activities did not differ between the three groups (Table 1).

In correlation analysis, TAS was negatively correlated with TOS, OSI, FPG, BMI, waist circumference, TG, LDLC, hsCRP, and SBP and DBP $(r=-0.384, P<0.001$; $r=-0.701, \quad P<0.001 ; r=-0.408, \quad P<0.001 ; r=$ $-0.414, \quad P<0.001 ; \quad r=-0.436, \quad P<0.001 ; \quad r=$ $-0.416, P<0.001 ; r=-0.237, P<0.05 ; r=-0.246$, $P<0.05 ; r=-0.467, P<0.001 ; r=-0.449, P<0.001$ respectively) and positively correlated with HDL-C $(r=0.281, P<0.01$; Table 2). OSI was found to be positively correlated with FPG, BMI, TG, LDL-C, hsCRP, waist circumference, SBP, and DBP $(r=0.281, P<0.01$; $r=0.329, P<0.01 ; \quad r=0.378, P<0.001 ; \quad r=0.319$, $P<0.01 ; \quad r=0.257, \quad P<0.05 ; \quad r=0.269, \quad P<0.01$; $r=0.377, P<0.001 ; r=0.409, P<0.001$ respectively) and negatively correlated with HDL-C $(r=-0.219$, $P<0.05$; Table 2). PON activity showed positive correlation with arylesterase activity and HDL-C levels $(r=0.351, \quad P<0.001$ and $\quad r=0.242, \quad P<0.05$ respectively).

In multiple regression analysis, $\mathrm{HDL}-\mathrm{C}$ was found to be the only variable affecting arylesterase and PON activities $(P<0.05$; Tables 3 and 4$)$.

Table 1 Clinical and metabolic parameters of the MetS, obese and control groups.

\begin{tabular}{|c|c|c|c|}
\hline & $\begin{array}{c}\text { MetS } \\
(n=40,3 \mathrm{M} \text { and } 37 \mathrm{~F})\end{array}$ & $\begin{array}{c}\text { Obese } \\
(n=30,3 \mathrm{M} \text { and } 27 \mathrm{~F})\end{array}$ & $\begin{array}{c}\text { Control } \\
(n=24,2 \mathrm{M} \text { and } 28 \mathrm{~F})\end{array}$ \\
\hline Age (years) & $34.7 \pm 7.2$ & $31.5 \pm 6.2$ & $33.1 \pm 7.8$ \\
\hline Body weight (kg) & $100.1 \pm 15.3^{\mathrm{b}, \mathrm{h}}$ & $90.7 \pm 19.1^{c}$ & $60.1 \pm 10.1$ \\
\hline BMI $\left(\mathrm{kg} / \mathrm{m}^{2}\right)$ & $40.3 \pm 6.2^{\mathrm{b}, \mathrm{e}}$ & $35.9 \pm 5.6^{c}$ & $23 \pm 3.3^{\mathrm{b}}$ \\
\hline Waist-C (cm) & $108.9+11^{a, b}$ & $99.4+12.9^{c}$ & $75.1+8.2$ \\
\hline $\mathrm{SBP}(\mathrm{mmHg})$ & $146.1 \pm 22.6^{\mathrm{a}, \mathrm{b}}$ & $118.5 \pm 12.5^{\mathrm{d}}$ & $98.3 \pm 28.2$ \\
\hline DBP $(\mathrm{mmHg})$ & $94.8 \pm 16.4^{\mathrm{a}, \mathrm{b}}$ & $80.0 \pm 9.0^{c}$ & $65.0 \pm 7.7$ \\
\hline $\mathrm{HDL}(\mathrm{mmol} / \mathrm{l})$ & $1.13 \pm 0.32^{\mathrm{b}}$ & $1.16 \pm 0.44^{d}$ & $1.45 \pm 0.14$ \\
\hline LDL $(\mathrm{mmol} / \mathrm{l})$ & $3.1 \pm 0.85^{\mathrm{b}}$ & $3.1 \pm 0.66^{c}$ & $2.3 \pm 0.84$ \\
\hline $\mathrm{TG}(\mathrm{mmol} / \mathrm{l})$ & $2.1 \pm 0.93^{\mathrm{a}, \mathrm{b}^{*}}$ & $1.12 \pm 0.42^{*}$ & $0.83 \pm 0.34^{*}$ \\
\hline $\mathrm{hsCRP}(\mathrm{mg} / \mathrm{l})$ & $0.87 \pm 0.7^{\mathrm{a}, \mathrm{b}^{*}}$ & $0.29 \pm 0.24^{\star}$ & $0.33 \pm 0.5^{\star}$ \\
\hline TOS ( $\mu \mathrm{mol} \mathrm{H}_{2} \mathrm{O}_{2}$ Equiv./l) & $13.1 \pm 2.9^{*}$ & $12.6 \pm 2.1^{*}$ & $12.1 \pm 2.3^{*}$ \\
\hline TAS (mmol Trolox Equiv./l) & $0.96 \pm 0.12^{\mathrm{b}, \mathrm{e}}$ & $1.1 \pm 0.18^{f}$ & $1.15 \pm 0.12$ \\
\hline OSI (arbitrary unit) & $13.77 \pm 3.3^{9}$ & $12.91 \pm 7.5$ & $10.62 \pm 5.0$ \\
\hline PON (U/I) & $194.5 \pm 113.2$ & $210.9 \pm 114.7$ & $209 \pm 125.8$ \\
\hline Arylesterase (U/I) & $137.9 \pm 30.25$ & $127.7 \pm 21.9$ & $127.1 \pm 26.3$ \\
\hline FPG $(\mathrm{mg} / \mathrm{dl})$ & $102.8 \pm 9.8^{\mathrm{b}, \mathrm{h}}$ & $98.7 \pm 7.6^{\dagger}$ & $91.2 \pm 8.5$ \\
\hline 120th minute plasma glucose & $122.3 \pm 33.7^{\mathrm{b}, \mathrm{e}}$ & $102.5 \pm 18.1$ & $95.2 \pm 19.1$ \\
\hline HOMA-IR & $4.9 \pm 2.5^{\mathrm{b}}$ & $3.4 \pm 1.4^{\mathrm{d}}$ & $1.4 \pm 0.3$ \\
\hline
\end{tabular}

BMI, body mass index; FPG, fasting plasma glucose; HDL/LDL, high-density lipoprotein/low-density lipoprotein; HOMA-IR, homeostasis model assessment insulin resistance index; hsCRP, high-sensitive C-reactive protein; MetS, metabolic syndrome; OSI, oxidative stress index; PON, paraoxonase; SBP/DBP, systolic blood pressure/diastolic blood pressure; TAS, total antioxidant status; TG, triglyceride; TOS, total oxidative status; Waist-C, waist circumference. ${ }^{*}$ Data in which non-parametric tests were used and expressed as median (range). $P<0.001$ : ${ }^{a}$ MetS versus obese; ${ }^{b}$ MetS versus control; ${ }^{c}$ obese versus

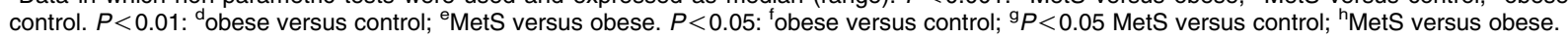


Table 2 Correlations between PON, arylesterase, and other clinical and metabolic parameters.

\begin{tabular}{|c|c|c|c|c|c|c|c|c|c|c|c|c|c|}
\hline & Arylesterase & TAS & TOS & OSI & FPG & BMI & TG & HDL-C & LDL-C & SBP & DBP & hsCRP & Waist-C \\
\hline \multicolumn{14}{|l|}{ PON } \\
\hline$r$ & 0.351 & -0.011 & 0.164 & 0.156 & 0.061 & -0.125 & -0.041 & 0.242 & 0.085 & 0.042 & -0.021 & 0.055 & -0.003 \\
\hline$P$ & $<0.001$ & 0.916 & 0.104 & 0.122 & 0.549 & 0.215 & 0.685 & 0.015 & 0.399 & 0.681 & 0.837 & 0.585 & 0.975 \\
\hline \multicolumn{14}{|c|}{ Arylesterase } \\
\hline$r$ & & -0.167 & 0.015 & 0.079 & 0.083 & -0.026 & 0.065 & 0.202 & 0.146 & 0.145 & 0.073 & 0.044 & 0.051 \\
\hline$P$ & & 0.097 & 0.886 & 0.433 & 0.414 & 0.794 & 0.522 & 0.044 & 0.148 & 0.150 & 0.471 & 0.664 & 0.617 \\
\hline \multicolumn{14}{|l|}{ TAS } \\
\hline$r$ & & & -0.384 & -0.701 & -0.408 & -0.414 & -0.416 & 0.281 & -0.237 & -0.467 & -0.449 & -0.246 & -0.436 \\
\hline \multirow{2}{*}{\multicolumn{14}{|c|}{$8.001<-0.001<-0.001<-0.001<-0.001$}} \\
\hline & & & & & & & & & & & & & \\
\hline$r$ & & & & 0.912 & 0.196 & 0.238 & 0.282 & -0.186 & 0.308 & 0.224 & 0.292 & 0.210 & 0.157 \\
\hline$P$ & & & & $<0.001$ & 0.050 & 0.017 & 0.005 & 0.065 & 0.002 & 0.025 & 0.003 & 0.036 & 0.120 \\
\hline \multicolumn{14}{|l|}{ OSI } \\
\hline$r$ & & & & & 0.281 & 0.329 & 0.378 & -0.219 & 0.319 & 0.377 & 0.409 & 0.257 & 0.269 \\
\hline$P$ & & & & & 0.005 & 0.001 & $<0.001$ & 0.029 & 0.001 & $<0.001$ & $<0.001$ & 0.010 & 0.007 \\
\hline \multicolumn{14}{|c|}{ 年 } \\
\hline$r$ & & & & & & 0.360 & 0.217 & -0.180 & 0.185 & 0.294 & 0.323 & 0.202 & 0.449 \\
\hline$P$ & & & & & & $<0.001$ & 0.030 & 0.074 & 0.065 & 0.003 & 0.001 & 0.044 & $<0.001$ \\
\hline \multicolumn{14}{|l|}{ BMI } \\
\hline$r$ & & & & & & & 0.418 & -0.423 & 0.321 & 0.390 & 0.475 & 0.428 & 0.706 \\
\hline$P$ & & & & & & & $<0.001$ & $<0.001$ & 0.001 & $<0.001$ & $<0.001$ & $<0.001$ & $<0.001$ \\
\hline \multicolumn{14}{|l|}{ TG } \\
\hline$r$ & & & & & & & & -0.246 & 0.166 & 0.396 & 0.402 & 0.268 & 0.446 \\
\hline$P$ & & & & & & & & 0.013 & 0.098 & $<0.001$ & $<0.001$ & 0.007 & $<0.001$ \\
\hline \multicolumn{14}{|l|}{ HDL-C } \\
\hline$r$ & & & & & & & & & -0.282 & -0.276 & -0.311 & -0.132 & -0.416 \\
\hline$P$ & & & & & & & & & 0.005 & 0.005 & 0.002 & 0.190 & $<0.001$ \\
\hline \multicolumn{14}{|l|}{ LDL-C } \\
\hline$r$ & & & & & & & & & & 0.253 & 0.299 & 0.087 & 0.348 \\
\hline$P$ & & & & & & & & & & 0.011 & 0.002 & 0.391 & $<0.001$ \\
\hline \multicolumn{14}{|l|}{ SBP } \\
\hline$r$ & & & & & & & & & & & 0.854 & 0.363 & 0.588 \\
\hline$P$ & & & & & & & & & & & $<0.001$ & $<0.001$ & $<0.001$ \\
\hline \multicolumn{14}{|l|}{ DBP } \\
\hline$r$ & & & & & & & & & & & & 0.359 & 0.607 \\
\hline$P$ & & & & & & & & & & & & $<0.001$ & $<0.001$ \\
\hline \multicolumn{14}{|l|}{ hsCRP } \\
\hline$r$ & & & & & & & & & & & & & 0.417 \\
\hline$P$ & & & & & & & & & & & & & $<0.001$ \\
\hline
\end{tabular}

BMI, body mass index; FPG, fasting plasma glucose; HDL/LDL, high-density lipoprotein/low-density lipoprotein; hsCRP, high-sensitive C-reactive protein; OSI, oxidative stress index; PON, paraoxonase; SBP/DBP, systolic blood pressure/diastolic blood pressure; TAS, total antioxidant status; TG, triglyceride; TOS, total oxidative status; Waist-C, waist circumference.

\section{Discussion}

Obesity and glucose intolerance, the components of MetS, often cause oxidative stress and are related with accelerated atherogenesis, as mentioned in several studies $(6,15-18)$. However, these studies did not exclude the effect of diabetes on oxidative stress in obesity and MetS. In this study, we analyzed TAS, TOS, and OSI as the overall markers of oxidative stress and hsCRP as a marker of subclinical inflammation and cardiovascular risk in obesity and MetS excluding diabetes. We also aimed to determine how PON-1, a more specific anti-oxidative enzyme with both PON and arylesterase activities, is affected in these groups.

We found that both hsCRP and TAS were altered in non-diabetic MetS and obese groups compared to controls, but oxidative state and subclinical inflammation were more severely affected in the MetS group when compared to the only obese group. This result is not surprising, since several metabolic alterations, such as hypertension, hypertriglyceridemia, low HDL-C levels, IGT, IFG, and insulin resistance, are frequently found in MetS when compared with obesity, as was seen in our morbidly obese MetS study sample. Since it is known that insulin resistance and adiposity, which are recognized as being related with altered oxidative stress, increase with increased BMI, this may explain the affected oxidant state in our morbidly obese MetS group $(19,20)$. These findings were concordant with previous studies, but the patient sample of our study was more uniform (i.e. age) than the previous study samples $(18,21)$. In one study sample, patient age was shown to be a critical point in the management of oxidative stress and the resultant inflammation (20). In the present study, our patients were younger than in the previous studies in which oxidative stress was evaluated in MetS 
Table 3 Multiple linear regression analysis of PON and other variables.

\begin{tabular}{lcc}
\hline & $\boldsymbol{\beta}$ Coefficients & $\boldsymbol{P}$ value \\
\hline BMI & -0.242 & 0.109 \\
Age & -0.086 & 0.420 \\
Waist circumference & 0.221 & 0.201 \\
Triglyceride & -0.059 & 0.616 \\
HDL-C & 0.292 & 0.010 \\
LDL-C & 0.128 & 0.245 \\
SBP & 0.135 & 0.491 \\
DBP & -0.189 & 0.345 \\
hSCRP & 0.069 & 0.544 \\
FPG & 0.061 & 0.591 \\
OSI & 0.214 & 0.075 \\
\hline
\end{tabular}

BMI, body mass index; FPG, fasting plasma glucose; HDL/LDL, high-density lipoprotein/low-density lipoprotein; hsCRP, high-sensitive C-reactive protein OSI, oxidative stress index; PON, paraoxonase; SBP/DBP, systolic blood pressure/diastolic blood pressure.

and obesity $(15,18,21)$. Thus, the data collected from this younger population may be accepted as a more clear suggestion of the altered oxidant state in MetS and obesity.

Another important factor that affects oxidative stress is the existence of altered carbohydrate metabolism. Diabetes has an important impact in increased oxidative stress and accelerated atherosclerosis (16, 17, 22). Subjects in the current study had diabetes-free MetS and obesity, so we can conclude that the altered oxidative stress existing in MetS and obesity is not solely related with the existence of diabetes. Existence of alterations in carbohydrate metabolism other than diabetes in our MetS group may have had an effect on the increased oxidative stress, but the severity of this may be lower when compared to diabetes. Lack of analysis of our MetS patients in subgroups with normal glucose tolerance, IFG, or IGT is a limitation of our study with respect to drawing a conclusion about the impact of prediabetes on this enzyme system when compared with diabetic patients. The negative correlation of TAS with FPG and positive correlation of OSI with FPG may be an evidence

Table 4 Multiple linear regression analysis of arylesterase and variables.

\begin{tabular}{lcc}
\hline & $\beta$ Coefficients & $\boldsymbol{P}$ \\
\hline BMI & -0.076 & 0.620 \\
Age & -0.066 & 0.548 \\
Waist circumference & 0.049 & 0.779 \\
TG & 0.063 & 0.597 \\
HDL-C & 0.300 & 0.010 \\
LDL-C & 0.197 & 0.081 \\
SBP & 0.285 & 0.156 \\
DBP & -0.170 & 0.405 \\
hSCRP & 0.007 & 0.955 \\
FPG & 0.076 & 0.510 \\
OSI & 0.010 & 0.937 \\
\hline
\end{tabular}

BMI, body mass index; FPG, fasting plasma glucose; HDL/LDL, high-density lipoprotein/low-density lipoprotein; hsCRP; high-sensitive C-reactive protein; OSI, oxidative stress index; SBP/DBP, systolic blood pressure/diastolic blood pressure; TG, triglyceride. of our latter conclusion about altered carbohydrate metabolism and oxidative stress. HDL is known to have the ability to protect LDL from oxidation and to have an impact on the decrease in adhesion molecule expression, the factors that may play a role in increased oxidative stress and hsCRP. Thus, low HDL-C might have been a responsible factor for the increased CRP and decreased TAS levels in our morbidly obese MetS and obese groups. The negative correlation between HDL-C and OSI and between TAS and hsCRP suggests this. In addition to these factors, existence of hypertension, which is well known as being related with oxidative stress and the progression of atherosclerosis, may also be a causal factor in the altered oxidant state in our MetS group. We also found OSI to be increased in the presence of high SBP and DBP in our study.

Although oxidative stress is accepted as an initiating factor in atherogenesis, it appears not to be the sole underlying mechanism $(23,24)$. Recent studies have shown that PON-1, a protective enzyme for LDL-C oxidation, is an important molecule in the pathogenesis of atherosclerosis (25). PON-1 has been studied in MetS and obesity in several studies in different study groups, and the results of these studies are conflicting (18, 26-28). Senti et al. showed that PON activity is lower in MetS compared to controls, and they suggested that the increased oxidative stress is related with the low PON activity (18). Although we found an increased inflammation and a limited antioxidant state in MetS in our study, our MetS group did not show any alteration in the PON-1 enzyme system. Age is known as an important determinant in PON activity (29). The control group was significantly younger than the MetS group in Senti et al.'s study; thus, the older age in the MetS group may explain the lower levels of PON activity in their study. They also included patients covering a wide age range. Several other factors, such as inclusion of diabetic patients and smokers, may also have affected the low PON-1 levels in the latter study $(30,31)$. Exclusion of such patients might explain the unaltered PON levels in our study.

The exact mechanism affecting low PON-1 levels in MetS is not clear. Although PON-1 activity has been found to be low in MetS, PON-1 codon 192 genotype distribution was found to be similar to that of the controls (18). It thus seems that low PON-1 levels in MetS may be an acquired condition due to the affected oxidant state. The non-smoker, non-diabetic, and younger population may explain our data of unaffected PON-1 activity in the MetS and obese groups. Since we did not determine PON-1 codon, we are limited in drawing a clear conclusion about this finding.

There are few studies about the relation between PON-1 activity and obesity in comparison with the normal population, so the data regarding how obesity affects PON-1 levels is not clear. It was found that HDL PON-1 activity in obese patients is lower than in controls in a relatively smaller obese population (26). 
In this study, low HDL PON-1 activity was found to be related with increased oxidative stress and is suggested to be the responsible factor in increased oxidative stress. Low PON-1 level may not initiate oxidative stress, but consumption of the enzyme due to increased oxidative stress may be another possibility. Since data regarding genetic determination of the enzyme is lacking, the initiating factor for this altered oxidative state in this obese group is not clear. We found PON and arylesterase activity in obese patients to be similar to that of the controls, despite an increased oxidative stress and subclinical inflammation. Since oxidative stress is the result of disturbed oxidant-antioxidant balance, this unchanged PON-1 activity in the state of increased oxidative balance may lead to oxidative stress and the resultant atherogenesis (32). Our results suggest that PON-1 activity is low or unchanged in non-diabetic obese patients despite increased oxidative stress, which may result in inadequate antioxidant defense.

In conclusion, oxidative stress and inflammation are increased in non-diabetic obese and MetS patient groups. Unchanged HDL-specific anti-atherogenic enzyme activities in obese and MetS patients may lead to an inadequate total antioxidant defense system and the resultant atherogenesis, even in those who are diabetes free.

\section{Declaration of interest}

The authors declare that there is no conflict of interest that could be perceived as prejudicing the impartiality of the research reported.

\section{Funding}

This research did not receive any specific grant from any funding agency in the public, commercial, or not-for-profit sector.

\section{References}

1 Draganov DI, Stetson PL, Watson CE, Billecke SS \& La Du BN. Rabbit serum paraoxonase 3 (PON3) is a high density lipoprotein-associated lactonase and protects low density lipoprotein against oxidation. Journal of Biological Chemistry 2000 275 33435-33442.

2 Getz GS \& Reardon CA. Paraoxonase, a cardioprotective enzyme: continuing issues. Current Opinion in Lipidology 200415 261-267.

3 Mackness M, Durrington PN \& Mackness B. Paraoxonase 1 activity, concentration and genotype in cardiovascular diseases. Current Opinion in Lipidology 200415 399-404.

4 Third Report of the National Cholesterol Education Program (NCEP). Expert panel on the detection, evaluation, and treatment of high blood cholesterol in adults (Adult Treatment Panel III). Final report. II Rationale for intervention. Circulation $2002 \mathbf{1 0 6}$ 3188-3190.

5 Misra A \& Khurana L. Obesity and the metabolic syndrome in developing countries. Journal of Clinical Endocrinology and Metabolism 200893 S9-S30.

$6 \mathrm{Kim} \mathrm{CH} \&$ Younossi ZM. Nonalcoholic fatty liver disease: a manifestation of the metabolic syndrome. Cleveland Clinic Journal of Medicine 200875 721-728.
7 Holvoet P. Relations between metabolic syndrome, oxidative stress and inflammation and cardiovascular disease. Verhandelingen - Koninklijke Academie Voor Geneeskunde Van Belgie 200870 193-219.

8 Erel 0 . A novel automated method to measure total antioxidant response against potent free radical reactions. Clinical Biochemistry 200437 112-119.

9 Erel 0 . A new automated colorimetric method for measuring total oxidant status. Clinical Biochemistry 200538 1103-1111.

10 Bolukbas C, Bolukbas FF, Horoz M, Aslan M, Celik H \& Erel O. Increased oxidative stress associated with the severity of the liver disease in various forms of hepatitis $\mathrm{B}$ virus infection. BMC Infectious Diseases 2005595.

11 Eckerson HW, Wyte MC \& La Du BN. The human serum paraoxonase/arylesterase polymorphism. American Journal of Human Genetics $1983351126-1138$.

12 Haagen L \& Brock A. A new automated method for phenotyping arylesterase (E.C.3.1.1.2.) based upon inhibition of enzymatic hydrolysis of 4-nitrophenyl acetate by phenyl acetate. European Journal of Clinical Chemistry and Clinical Biochemistry 199230 391-395.

13 Matthews DR, Hosker JP, Rudenski AS, Naylor BA, Treacher DF \& Turner RC. Homeostasis model assessment: insulin resistance and beta-cell function from fasting plasma glucose and insulin concentrations in man. Diabetologia $19852 \mathbf{2 8} 412-419$.

14 Radikova Z, Koska J, Huckova M, Ksinatova L, Imrich R, Vigas M, Trnovec T, Langer P, Sebokova E \& Klimes I. Insulin sensitivity indices: a proposal of cut-off points for simple identification of insulin-resistant subjects. Experimental and Clinical Endocrinology and Diabetes $2006 \mathbf{1 1 4} 249-256$.

15 Hansel B, Giral P, Nobecourt E, Chantepie S, Bruckert E, Chapman MJ \& Kontush A. Metabolic syndrome is associated with elevated oxidative stress and dysfunctional dense highdensity lipoprotein particles displaying impaired antioxidative activity. Journal of Clinical Endocrinology and Metabolism 200489 4963-4971.

16 Eschwege E. The dysmetabolic syndrome, insulin resistance and increased cardiovascular (CV) morbidity and mortality in type 2 diabetes: aetiological factors in the development of $\mathrm{CV}$ complications. Diabetes E Metabolism 200329 6S19-6S27.

17 Rosen P, Nawroth PP, King G, Moller W, Tritschler HJ \& Packer L. The role of oxidative stress in the onset and progression of diabetes and its complications: a summary of a Congress Series sponsored by UNESCO-MCBN, the American Diabetes Association and the German Diabetes Society. Diabetes/Metabolism Research and Reviews 200117 189-212.

18 Senti M, Tomas M, Fito M, Weinbrenner T, Covas MI, Sala J, Masia R \& Marrugat J. Antioxidant paraoxonase 1 activity in the metabolic syndrome. Journal of Clinical Endocrinology and Metabolism $2003 \mathbf{8 8} 5422-5426$.

19 Roberts CK \& Sindhu KK. Oxidative stress and metabolic syndrome. Life Sciences $2009 \mathbf{8 4} 705-712$.

20 Vincent HK, Innes KE \& Vincent KR. Oxidative stress and potential interventions to reduce oxidative stress in overweight and obesity. Diabetes, Obesity and Metabolism 20079 813-839.

21 Demircan N, Gurel A, Armutcu F, Unalalacak M, Aktunc E \& Atmaca $\mathrm{H}$. The evaluation of serum cystatin $\mathrm{C}$, malondialdehyde, and total antioxidant status in patients with metabolic syndrome. Medical Science Monitor 2008 14 97-101.

22 Giugliano D, Ceriello A \& Paolisso G. Oxidative stress and diabetic vascular complications. Diabetes Care 199619 257-267.

23 Madamanchi NR, Vendrov A \& Runge MS. Oxidative stress and vascular disease. Arteriosclerosis, Thrombosis, and Vascular Biology $20052529-38$.

24 Lubos E, Handy DE \& Loscalzo J. Role of oxidative stress and nitric oxide in atherothrombosis. Frontiers in Bioscience 200813 5323-5344.

25 Kabaroglu C, Mutaf I, Boydak B, Ozmen D, Habif S, Erdener D, Parildar Z \& Bayindir O. Association between serum paraoxonase activity and oxidative stress in acute coronary syndromes. Acta Cardiologica 200459 606-611. 
26 Ferretti G, Bacchetti T, Moroni C, Savino S, Liuzzi A, Balzoa F \& Bicchiega V. Paraoxonase activity in high-density lipoproteins: a comparison between healthy and obese females. Journal of Clinical Endocrinology and Metabolism $2005901728-1733$.

27 Paragh G, Seres I, Harangi M, Erdei A, Audikovszky M, Debreczeni L, Kovacsay A, Illyes L \& Pados G. Ciprofibrate increases paraoxonase activity in patients with metabolic syndrome. British Journal of Clinical Pharmacology 200661 694-701.

28 Audikovszky M, Pados G, Seres I, Harangi M, Fulop P, Katona E, Illyes L, Winkler G, Katona EM \& Paragh G. Orlistat increases serum paraoxonase activity in obese patients. Nutrition, Metabolism, and Cardiovascular Diseases 200717 268-273.

29 Senti M, Tomas M, Vila J, Marrugat J, Elosua R, Sala J \& Masia R. Relationship of age-related myocardial infarction risk and Gln/Arg 192 variants of the human paraoxonase1 gene: the REGICOR study. Atherosclerosis 2001156 443-449.
30 Mackness B, Mackness MI, Arrol S, Turkie W, Julier K, Abuasha B, Miller JE, Boulton AJM \& Durrington PN. Serum paraoxonase (PON1) 55 and 192 polymorphism and paraoxonase activity and concentration in non-insulin dependent diabetes mellitus. Atherosclerosis 1998139 341-349.

31 Senti M, Tomas M, Anglada R, Elosua R, Marrugat J, Covas MI \& Fitoo M. Interrelationship of smoking, paraoxonase activity and leisure-time physical activity: a population-based study. European Journal of Internal Medicine 2003 14 178-184.

32 Jones DP. Redefining oxidative stress. Antioxidants and Redox Signaling 20068 1865-1879.

Received 16 December 2009

Accepted 17 December 2009 